\title{
Filtration mit kompressiblen Kuchen: Effiziente Konzepte für eine anspruchsvolle Trennaufgabe
}

CARINA MARIA ALLES * UND HARALD ANLAUF

Herrn Prof. Dr.-Ing. WERnER STAHL zum 65. Geburtstag

Die Filtration mit kompressiblen $\mathrm{Ku}$ chen ist eine anspruchsvolle Trennaufgabe, die nicht selten für den Erfolg oder Misserfolg eines Verfahrens ausschlaggebend ist. Methoden, die sich zur Prozessauslegung bei inkompressiblen Filterkuchen bewährt haben, können nicht ohne weiteres übernommen werden. Statt dessen sind neue Konzepte gefragt, die auf die Besonderheiten kompressiblen Materialverhaltens eingehen. In diesem Beitrag werden effiziente Prozessstrategien für die Filtration mit kompressiblen Kuchen vorgestellt. Dazu wurde ein physikalisch-mathematisches Modell in eine Prozesssimulation umgesetzt, mit der bei minimiertem experimentellen Aufwand Haufwerksstrukturen und Trennergebnisse bei verschiedenen $\mathrm{Be}$ triebszuständen im Voraus berechnet werden können. Zur Bestimmung der erforderlichen Materialdaten sind standardisierte Versuchsmethoden an einer neuen Laborapparatur entwickelt worden.
Efficient Process Strategies for Compressible Cake Filtration

Compressible cake filtration is a challenging and often critical task in many industrial processes. Conventional design methods are not always appropriate as they fail to address the specifics of compressible structures. Efficient strategies for compressible cake filtration will be presented. The guidelines for process design and optimization are the outcome of experimental investigations, utilizing an innovative compression-permeability-filter cell, and a process simulation based on a rigorous model of compressible cake formation and consolidation.

\section{Einleitung}

Die kuchenbildende Filtration ist eine bewährte Methode zur Abscheidung suspendierter Feststoffpartikel aus einer Flüssigkeit. Sie beruht darauf, dass die Flüssigkeit unter dem Einfluss eines Druckgradienten durch ein Filtermedium strömt, an dem der Feststoff zurückgehalten wird. Die abgelagerten Partikel bilden den Filterkuchen, an dessen Oberfläche der Feststoff im weiteren Verlauf abgeschieden wird. Die Struktur des Filterkuchens kann durch seine Porosität und seinen Durchströmungswiderstand charakterisiert werden. Eine effiziente Filtration zeichnet sich dadurch aus, dass der Filterkuchen schnell aufgebaut wird (geringer Widerstand) und wenig Flüssigkeit enthält (geringe Porosität).

Filterkuchen werden häufig als starre Packungen angesehen, deren Struktur unbeeinflusst von den Prozessbedingungen bleibt. Solche inkompressiblen Kuchen sind jedoch eher die Ausnahme als die Regel. Tatsächlich sind die meisten Filterkuchen mehr oder minder kompressibel, d.h. sie lassen sich unter Druckbeanspruchung kompaktie-

* Dr-Ing C M ALLES (E-mail: carina alles@ usa.dupont.com), DuPont Engineering Technology, E.I. du Pont de Nemours and Company; 1007 Market St, B9458; Wilmington, DE 19898, USA

Dr.-Ing. H. ANLAUF, (E-mail: harald. anlauf@mvm.uni-karlsruhe.de),Institut für Mechanische Verfahrenstechnik und Mechanik, Universität Karlsruhe (TH) D-76128 Karlsruhe, Germany. ren. Porosität und Durchströmungswiderstand sind dann vom Filtrationsdruck abhängig. Suspensionen, aus denen besonders kompressible Kuchen entstehen, fallen in großen Mengen an, beispielsweise bei der Produktion von Pigmenten und anderen feindispersen Feststoffen, bei der Verarbeitung von Lebensmitteln, in der Biotechnologie, in der Abwasserreinigung und in der Aufbereitung von Feinstfraktionen mineralischer Rohstoffe.

Das kompressible Materialverhalten kann Probleme aufwerfen, die eine effiziente Filtration erschweren, wenn nicht sogar vereiteln [1]:

- Während der Kuchenbildung ist die Haufwerksstruktur inhomogen. Am Filtermedium liegt eine dünne, stark kompaktierte Grundschicht vor (s. Abschnitt 2.2), während der Rest des Kuchens hoch porös bleibt und viel Flüssigkeit bindet.

- Die inhomogene Kuchenstruktur erschwert Handhabung, Transport oder Trocknung des Kuchens.

- Kuchenbildung, Waschung und Pressung nehmen viel Zeit in Anspruch, weil die verdichtete Grundschicht wie ein Flaschenhals das Abströmen des Filtrats behindert.

- Die hoch porösen Bereiche außerhalb der Grundschicht binden viel Flüssigkeit. Deshalb werden nach der $\mathrm{Ku}-$ chenbildung selten zufriedenstellende Restfeuchten erreicht.

- Zur weitergehenden Absenkung der Restfeuchte scheidet die Gasdifferenzdruckentfeuchtung in der Regel aus, weil kompressible Filterkuchen eine ausgeprägte Nei- 
gung zur Bildung von Schrumpfrissen zeigen [2]. Für Filterkuchen, die aus sehr feinen Partikeln $(<1 \mu \mathrm{m})$ aufgebaut sind, ist diese Entfeuchtungsmethode aufgrund der hohen kapillaren Eintrittsdrücke ohnehin nicht interessant.

- Besonders kompressible Kuchen entstehen aus Suspensionen, die einen hohen Anteil feiner Partikel $(<10 \mu \mathrm{m})$ enthalten. In solchen Suspensionen haben physikochemische Suspensionseigenschaften wie $\mathrm{pH}$-Wert und Ionengehalt starken Einfluss auf die Agglomeration der Partikel und damit auf die Filterkuchenstruktur (s. Abschnitt 2.1). Schwankungen der Suspensioneigenschaften wirken sich daher unmittelbar auf das Trennergebnis aus [3-5]. Die Kontrolle solcher Prozesse ist äußerst problematisch.

Leitlinien zu effizienten Prozessstrategien für die Filtration mit kompressiblen Kuchen werden in der Praxis vermisst. Der Einsatz vereinfachter Konzepte für inkompressible Kuchen führt nicht selten zu Unsicherheiten und Fehlern in der Prozessauslegung.

\section{Kompressibles Materialverhalten 2.1 Mechanismen}

Die Ursachen kompressiblen Verhaltens von Haufwerken sind vielfältig und werden in erster Linie phänomenologisch beschrieben [2, 6, 7]. Allgemein gilt, dass kompressible Haufwerke nicht zwangsläufig aus kompressiblen Partikeln bestehen. Abb. 1 veranschaulicht die verschiedenen Mechanismen, die für Kompressibilität verantwortlich gemacht werden. In realen Haufwerken überlagern sich diese $\mathrm{Me}-$ chanismen häufig.

Abbildung 1.

Ursachen kompressiblen Materialverhaltens; a) Umlagerung von Partikeln; b) Wechselwirkungen zwischen feinen Partikeln; c) Deformation von Partikeln; d) Überschreiten der Materialfestigkeit.

a) Umlagerung von Partikeln
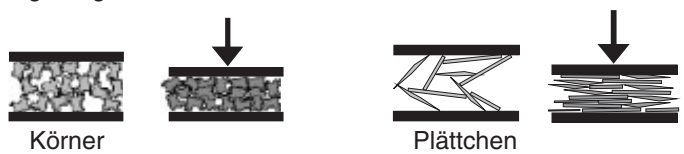

b) Wechselwirkungen zwischen feinen Partikeln
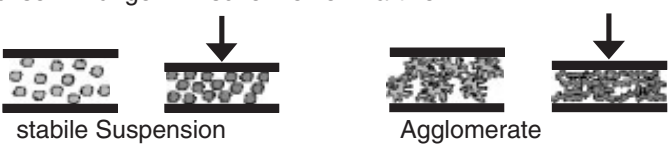

c) Deformation von Partikeln
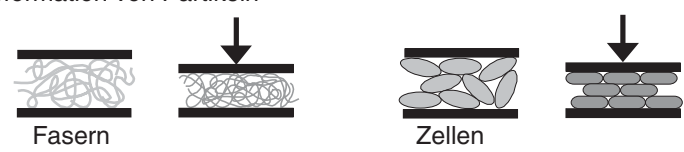

d) Überschreiten der Materialfestigkeit
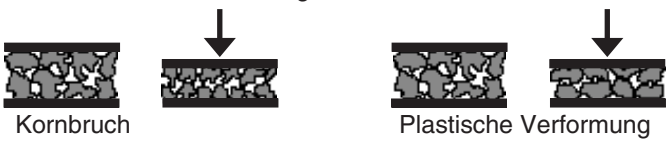

Die Strukturen, die sich in Filterkuchen spontan bilden, entsprechen nur in Ausnahmefällen der dichtesten Packung. In lockeren Verbänden können sich Partikel unter mechanischer Belastung zu einer dichteren Packung umlagern [2]. Besonders ausgeprägt ist diese Form der Kompressibilität bei plättchenförmigen Partikeln, die von einer lockeren Kartenhausstruktur zu einer dachziegelartig eng gepackten Struktur übergehen (s. Abb. 1a).

In feindispersen Systemen gewinnen Oberflächenkräfte gegenüber Massen- und Trägheitskräften an Bedeutung [3 - 5]. Je nach dem, ob die Grenzflächeneffekte zu Anziehung oder Abstoßung zwischen den Partikeln führen, bilden die Partikel Agglomerate oder liegen vereinzelt in der dann stabil genannten Suspension vor. Als Faustregel gilt, dass Agglomerate hochporöse, durchlässige Filterkuchen bilden, die schon bei geringen Drücken stark kompaktiert werden. Filterkuchen, die aus stabilen Suspensionen gebildet werden, sind in der Regel kompakter und weniger durchlässig. Sie sind nur dann kompressibel, wenn die abstoßenden Kräfte durch die mechanische Belastung überwunden werden können (s. Abb. 1b).

Wenn die Partikel selbst sich unter Druckbeanspruchung verformen, führt dies unmittelbar zu kompressiblen Haufwerken (s. Abb. 1c).

Erst bei sehr hohen Drücken, die für technische Anwendungen in der Fest/Flüssig-Trennung selten in Frage kommen, werden die Grenzen der Materialfestigkeit überschritten, und es kommt zu Kornbruch oder plastischem Fließen [2]. Diese Vorgänge sind in Abb. 1d dargestellt.

Haufwerke, deren Struktur allein von der mechanischen Belastung abhängt, werden als ideal kompressibel bezeichnet.

\subsection{Filterkuchenstruktur}

In Prozessen der Fest/Flüssig-Trennung werden Haufwerke durchströmt. Daraus resultiert ein Druckverlust in der Flüssigkeit, der vom Feststoffgerüst aufgenommen wird. Ein inkompressibles Haufwerk bleibt dabei unverändert.

Kompressible Haufwerke werden hingegen bei Durchströmung kompaktiert. Je höher der lokale Feststoffgerüstdruck, um so niedriger ist die lokale Porosität und um so höher ist der lokale Durchströmungswiderstand. Bei der Filtration unter reinem Differenzdruck und ohne Massenkräfte fällt der hydraulische Druck stetig in Richtung Filtermedium ab. Der Feststoffgerüstdruck nimmt in Gegenrichtung zu und erreicht unmittelbar am Filtermedium seinen Höchstwert. Dementsprechend ist dort der Kuchen am stärksten kompaktiert, der lokale Widerstand und damit der lokale Druckgradient sind am größten. Im restlichen $\mathrm{Ku}$ chen sind die Druckgradienten schwach, und die Struktur ist kaum verdichtet (s. Abb. 2). Bei hoch kompressiblen Filterkuchen ist die Grundschichtverdichtung extrem: Auf dem Filtermedium liegt eine lederartige, nahezu undurchlässige Haut (engl. skin effect), während die darüber liegenden Schichten einer eingedickten Suspension ähneln [8]. Der Übergang zwischen Kuchen und Suspension ist dann kaum feststellbar. 
Abbildung 2.

Grundschichtverdichtung in kompressiblen Filterkuchen.

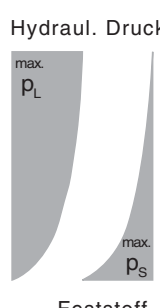

gerüstdruck

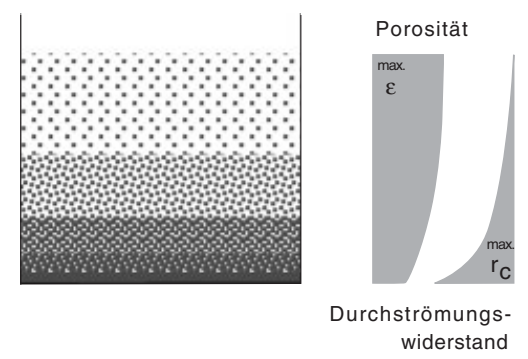

3 Modelle für die Fest/Flüssig-Trennung mit kompressiblen Haufwerken

3.1 Globale Betrachtung der Kuchenbildung

Zur Auslegung von Filtrationsprozessen in der Praxis ist die globale Betrachtungsweise am weitesten verbreitet [9-13]. Dabei wird der Kuchen, ungeachtet von Strukturunterschieden im Innern, als quasi homogene Einheit aufgefasst.

Im Mittelpunkt steht die sogenannte Grundgleichung der kuchenbildenden Filtration (s. Gl. (1)), die in der Praxis häufig zur Prozessauslegung herangezogen wird [14, 15]. Zwar gilt diese Beziehung zunächst nur für inkompressible Haufwerke, sie wird aber häufig auf den kompressiblen Fall übertragen, in dem die inhomogene Kuchenstruktur durch Mittelwerte der Porosität $\bar{\varepsilon}$ und des Widerstandes $\bar{\alpha}$ beschrieben wird [9] ${ }^{1)}$ :

$\frac{\mathrm{d} v}{\mathrm{~d} t}=\frac{\Delta p}{\eta\left(R_{\mathrm{M}}+\bar{\alpha}(1-\bar{\varepsilon}) \rho_{\mathrm{S}} \kappa v\right)}$

Der Widerstand des Kuchens im Nenner der Gleichung wird als Produkt eines materialtypischen Widerstandskennwertes und der Kuchenhöhe $h_{\mathrm{C}}$ bzw. der Feststoffmasse $w_{S, C}$ des Kuchens angegeben (s. Gl. (2)). Gebräuchliche Kennwerte sind der längenbezogene Widerstand $r_{\mathrm{C}}$, die längenbezogene Permeabilität $P_{\mathrm{C}}$ und der massenbezogene Widerstand $\alpha$. Der Konzentrationsbeiwert $\kappa$ ist der Proportionalitätsfaktor, der Kuchenhöhe $h_{\mathrm{C}}$ und flächenspezifisches Filtratvolumen $v$ verknüpft.

$R_{\mathrm{C}}=r_{\mathrm{C}} h_{\mathrm{C}}=\frac{h_{\mathrm{C}}}{P_{\mathrm{C}}}=\alpha w_{\mathrm{S}, \mathrm{C}}=\alpha(1-\varepsilon) \rho_{\mathrm{S}} h_{\mathrm{C}}=\alpha(1-\varepsilon) \rho_{\mathrm{S}} \kappa v$

Für die Abhängigkeit der mittleren Porosität und des mittleren Durchströmungswiderstandes von der Druckdifferenz am Filterkuchen $\Delta p_{\mathrm{C}}$ sind verschiedene empirische Ansätze der Form $\bar{\varepsilon}\left(\Delta p_{\mathrm{c}}\right)$ und $\bar{\alpha}\left(\Delta p_{\mathrm{c}}\right)$ bekannt [11, 13, $16,17]$, wie zum Beispiel:

$(1-\bar{\varepsilon})=\left(1-\overline{\varepsilon_{0}}\right)\left(1+\frac{\Delta p_{\mathrm{C}}}{\Delta p_{0}}\right)^{\mathrm{N}_{\varepsilon}}$

$\bar{\alpha}=\overline{\alpha_{0}}\left(1+\frac{\Delta p_{\mathrm{C}}}{\Delta p_{0}}\right)^{\mathrm{N}_{\alpha}}$

Der Exponent $\mathrm{N}_{\alpha}$ aus Gl. (4) wird globale Kompressibilität genannt und dient als Anhaltspunkt zur Beurteilung des kompressiblen Materialverhaltens. Inkompres-

1) Eine Zusammenstellung der Formelzeichen befindet sich am Schluss des Beitrags. sible Filterkuchen sind durch den Exponenten $\mathrm{N}_{\alpha}=0$ gekennzeichnet, für stark kompressible Materialien strebt der Exponent $\mathrm{N}_{\alpha}$ gegen Eins [1, 17].

Ähnliche Potenzgesetze wie für den massenspezifischen Widerstand $\bar{\alpha}$ können auch für den höhenspezifischen Widerstand $\bar{r}_{\mathrm{c}}$ formuliert werden. Weil bei einer Druckerhöhung die Kuchenfeststoffmasse konstant bleibt, die Höhe kompressibler Kuchen sich aber vermindert, reagiert der höhenspezifische Widerstand empfindlicher auf Drucksteigerungen als der massenspezifische Widerstand. Nach den Gln. (3) und (4) ergibt sich für den höhenspezifischen Widerstand $\bar{r}_{\mathrm{c}}$ ein Exponent mit dem Wert $\mathrm{N}_{\varepsilon}+\mathrm{N}_{\alpha}$, der im Fall stark kompressibler Materialien den Wert Eins übersteigen kann.

Die globale Betrachtung der Filtration liefert nur dann vertrauenswürdige Werte zur Auslegung, wenn im Laborversuch die Prozessbedingungen der technischen Umsetzung möglichst genau abgebildet werden können. Eine veränderte Prozessführung erfordert neue Versuchsreihen. Variantenstudien sind darum mit großem experimentellen Aufwand verbunden. Ein weiterer Nachteil der globalen Betrachtung besteht darin, dass sie keinerlei Informationen über die innere Struktur der Filterkuchen liefert. Besonderheiten wie die Grundschichtverdichtung bei der Filtration mit kompressiblen Kuchen können somit nicht erfasst werden. Schließlich soll noch einmal betont werden, dass dieser Ansatz allein für die Kuchenbildung gilt, nicht für die Pressfiltration oder andere Verfahren der Fest/Flüssig-Trennung.

\subsection{Lokale Betrachtung von Fest/Flüssig- Trennprozessen}

Im Gegensatz zur globalen Betrachtung gibt die lokale Betrachtung Aufschluss über die Vorgänge, die während eines Fest/Flüssig-Prozesses im Inneren des Haufwerkes ablaufen. Dazu wird das betrachtete Haufwerk in dünne, quasi homogene Schichten parallel zum Filtermedium zerlegt, für die neben einem Durchströmungsgesetz Massen- und Kräftebilanzen aufgestellt werden.

Die Randbedingungen am Filtertuch und der Haufwerksoberfläche richten sich nach dem ausgewählten Prozess. Diese Herangehensweise ist allgemein zur physikalisch-mathematischen Beschreibung durchströmter Haufwerke geeignet. Sie ist deshalb auf verschiedene Fest/Flüssig-Trennprozesse wie Kuchenbildung, Sedimentation und Konsolidierung übertragbar und auch auf die Überlagerung der Grundvorgänge [17].

Im folgenden stehen die grundlegenden Arbeiten von FRANK TILLER (University of Houston, USA) im Mittelpunkt, der sein wissenschaftliches Lebenswerk der Fest/ Flüssig-Trennung mit kompressiblen Haufwerken gewidmet hat (s. [1] für ein umfassendes Literaturverzeichnis). Die wichtigsten Elemente dieses physikalisch-mathematischen Modells sind neben den allgemeinen Kräfte- und Massenbilanzen ein spezielles Durchströmungsgesetz [18], das die Relativbewegung zwischen Feststoff und Flüssigkeit berücksichtigt, und Materialgesetze, die den Einfluss des Feststoffgerüstdruckes auf die Haufwerksstruktur erfassen. Einzelheiten des Modells sind in [1] dargestellt. 
TILLER geht von ideal kompressiblen Haufwerken aus, deren Verformung voll plastisch sei, d.h. bei abnehmender Belastung soll die ursprüngliche Kompaktierung erhalten bleiben. Verschiedene empirische Materialgesetze stehen zur Auswahl, wie zum Beispiel:

$(1-\varepsilon)=\left(1-\varepsilon_{0}\right)\left(1+\frac{p_{\mathrm{S}}}{p_{\mathrm{S}, 0}}\right)^{\beta}$

und

$\alpha=\alpha_{0}\left(1+\frac{p_{\mathrm{S}}}{p_{\mathrm{S}, 0}}\right)^{\mathrm{n}}$

Die Summe $\mathrm{n}+\beta$ der Exponenten aus den Gln. (5) und (6) wird als charakteristische Größe zur Beurteilung des Materialverhaltens herangezogen [17]. Während inkompressible Materialien das Kriterium $(\mathrm{n}+\beta)=0$ erfüllen, werden Materialien mit $(\mathrm{n}+\beta)$ zwischen Eins und Zwei als hoch kompresssibel und Materialien mit $(n+\beta)$ über Zwei als super kompressibel eingestuft.

Aus den Grundgleichungen für die einzelnen $\mathrm{Ku}$ chenschichten, mit den Massenbilanzen für das Gesamtsystem und prozessspezifischen Randbedingungen ergibt sich ein System gekoppelter Gleichungen, das in dieser Form nur numerisch gelöst werden kann.

Die gebräuchlichste Vereinfachung zur analytischen Lösung der Gleichungen besteht darin, den Feststofffluss im Haufwerk zu vernachlässigen. Damit werden für einen vorgegebenen Druckverlust am Kuchen bei bekannten Materialgesetzen die Mittelwerte von Porosität und Widerstand berechenbar, die dann in die Grundgleichung der kuchenbildenden Filtration (Gl. (1)) eingesetzt werden können. So wird der Bogen von der lokalen zur globalen Betrachtung geschlagen. Dieses vereinfachte Modell nach TILLER wird oft zu einer groben, orientierenden Prozessauslegung eingesetzt $[11,13,19,20]$.

Die Vernachlässigung des Feststoffstroms ist nur bei der kuchenbildenden Filtration aus stark verdünnten Suspensionen angemessen, in denen der Feststoffanteil weniger als ca. 50 \% des Feststoffanteils in der obersten Kuchenschicht beträgt [18]. Ansonsten kann diese Vereinfachung insbesondere bei kurzen Filtrationszeiten zu starken Abweichungen vom tatsächlichen Ergebnis führen. Bei der Pressfiltration beruht die Flüssigkeitsströmung allein auf der Verschiebung von Feststoff im Kuchen; in diesem Fall ist es nicht zulässig, den Feststoffstrom unberücksichtigt zu lassen. Eine Modellierung, die Kuchenbildung und Pressung umfassen soll, muss daher auf diese Vereinfachung verzichten.

\subsection{Weitere Ansätze zur Beschreibung der Bildung und Konsolidierung kompressibler Haufwerke}

Eine Durchströmung kompressibler Packungen tritt in vielen technischen und natürlichen Prozessen auf. Dementsprechend finden sich Modelle zur Beschreibung dieses Grundvorganges in verschiedenen Disziplinen über die mechanische Verfahrenstechnik hinaus, wie z.B. in der Umwelttechnik, der Bodenmechanik oder der Lebensmittelwis- senschaft. Die Vielfalt der Themengebiete spiegelt sich in den unterschiedlichen Konzepten zur physikalisch-mathematischen Modellierung wider. Die Mehrzahl der Modelle geht von einer lokalen Betrachtung aus. Die Lösung der Gleichungssysteme verlangt in der Regel Vereinfachungen, die den Geltungsbereich der Modelle eingrenzen. In [1] werden die Arbeiten verschiedener Forschungsgruppen gegenübergestellt und ihre Relevanz für die Prozessauslegung kritisch beleuchtet.

Für die Umsetzung der Modelle in die Praxis ist entscheidend, wie die Parameter der Materialgesetze bestimmt werden sollen. Dazu gibt es zwei grundsätzlich verschiedene Konzepte.

Die Kompressions-Permeabilitäts-Zelle (C-PZelle, aus dem Englischen: compression-permeability) hat sich als Standardapparat durchgesetzt [16, 21, 22]. Hinzu kommen ähnliche Apparate, die sich in der Rheologie bewährt haben [23]. Die Materialparameter müssen nur einmal bestimmt werden, um dann auf unterschiedliche Fest/ Flüssig-Trennprozesse übertragen werden zu können. Die Prozessauslegung wird dadurch einfach und effizient.

Die Übertragbarkeit von Kompressions-Permeabilitäts-Daten auf Filtrationsprozesse ist nicht unumstritten [21]. Das Hauptproblem der Wandreibung lässt sich bei geeigneter Gestaltung der C-P-Zelle minimieren [24]. Mit Korrekturfunktionen kann auf den Idealfall der reibungsfreien Zelle rückgeschlossen werden [7].

Einige Kritiker der C-P-Methode favorisieren die Alternative, Informationen zur Kuchenstruktur unmittelbar aus dem Filtrationsversuch zu gewinnen [25, 26]. Zur Messung der lokalen Porositäten oder des lokalen hydraulischen Drucks sind aufwändige Messsonden erforderlich. Die Signalauswertung ist bei inhomogenen Kuchen problematisch. Die räumliche Auflösung der Messungen liegt in der Regel im Bereich einiger Millimeter. Die Untersuchungen bleiben damit auf dicke Filterkuchen und sehr lange Filtrationszeiten beschränkt. Einflüsse durch Sedimentation und Nachrücken von Feinkorn können dann nicht sicher ausgeschlossen werden. Darüber hinaus besteht die Gefahr, dass die empfindliche Kuchenstruktur durch die Sonden gestört wird.

Der schwerwiegendste Nachteil dieser Modelle besteht darin, dass mit ihnen lediglich bereits durchgeführte Experimente nachvollzogen werden können. Die Vorausberechnung des Filtrationsergebnisses bei veränderten Prozessbedingungen ist nicht möglich. Daher sind sie für die Prozessauslegung wenig geeignet.

\section{Simulationsprogramm}

Der lokale Ansatz nach TILLER wurde ohne weitere Vereinfachungen als Grundlage der physikalisch-mathematischen Modellierung der Bildung und Konsolidierung kompressibler Haufwerke gewählt. Der Algorithmus zur numerischen Lösung der Gleichungssysteme wurde in ein Computerprogramm zur Simulation von Filtration, Sedimentation und Pressung umgesetzt [1]. Wenn Material- und Prozessparameter vorgegeben werden, berechnet das Programm zu je- 
dem Zeitpunkt globale Größen wie das Filtratvolumen, die Haufwerkshöhe, den Konsolidierungsgrad und die Mittelwerte von Porosität und Durchströmungswiderstand, ebenso lokale Größen wie hydraulischen Druck und Feststoffgerüstdruck, Porosität und Durchströmungswiderstand. Damit können die zeitabhängigen Strukturänderungen während Kuchenbildung und -konsolidierung dargestellt werden, die direkten Messungen kaum zugänglich sind.

Die globalen Größen, die das Simulationsprogramm berechnet, können direkt zur Prozessauslegung verwendet werden. Damit wird die Möglichkeit eröffnet, bei minimiertem experimentellen Aufwand ausgewählte Verfahren bei verschiedenen Betriebszuständen zu analysieren. Auf dieser Grundlage kann entschieden werden, wie ein bestehendes Verfahren optimiert werden kann, wo die verfahrenstechnischen Grenzen liegen und wo innovative Verfahrenskonzepte ansetzen sollten.

Ein Vergleich zwischen experimentellen Daten zur Kuchenbildung und entsprechenden Simulationsdaten ergab eine gute qualitative Übereinstimmung [1]. Die Simulation ist also für den prinzipiellen Verfahrensentwurf und die Apparateauswahl geeignet, die genaue Dimensionierung des Filters sollte aber im Einzelfall durch ergänzende Filtrationsexperimente im interessanten Druckbereich abgesichert werden.

\section{Experimentelle Methoden und Materialien}

Zur Erfassung des Materialverhaltens sind Experimente nach wie vor unumgänglich. Die experimentellen Methoden der Laborfiltration [15] müssen an die Besonderheiten kompressibler Filterkuchen angepasst werden.

In der neu entwickelten Laborapparatur sind eine Kompressions-Permeabilitäts-Zelle (C-P-Zelle) und eine Drucknutsche zur Filtration in Tandem-Anordnung als C-P-F-Zelle aufgebaut (s. Abb. 3). Beide Komponenten sind für Drücke bis zu 16 bar ausgelegt, was für die Auslegung gebräuchlicher Filterapparate hinreichend ist. Die Tandem-Anordnung ermöglicht eine sehr flexible Versuchsführung, weil die Grundversuchstypen Kuchenbildung, Waschung und Durchströmung auf vielfältige Weise miteinander kombiniert werden können.

Bei der Kuchenbildung mit konstantem oder stufenweise ansteigendem Druck ergibt die Analyse des zeitabhängigen Filtratstroms die Widerstände des Filtermediums und des Kuchens. Dabei ist es wichtig, die Anfangsphase der Filtration verzögerungsfrei in kurzen Zeitintervallen zu erfassen, ansonsten treten vor allem beim Mediumwiderstand große Fehler auf [1]. Nach dem Versuchsende werden die Höhe und die mittlere Porosität des entlasteten Kuchens gemessen. Die Ergebnisse der Kuchenbildung können für einfache Auslegungen gemäß der globalen Betrachtung verwendet werden.

Zur Darstellung der Ergebnisse ist es ratsam, den Quotienten aus der differentiellen Zeit $\mathrm{d} t$ und dem differentiellen flächenbezogenen Filtratvolumen $d v$ mit dem Filtrationsdruck $\Delta p$ und der Flüssigkeitsviskosität $\eta$ zu normieren
Abbildung 3.

Schnittdarstellung der C-P-F Zelle, mit 1 - Filterkuchen, 2 - Filtermedium, 3 - Verteilerplatte, 4 - Kuchenbildungsring, 5 - Filtratablauf, 6 - Deckel mit Schauglas, 7 - Presskolben, 8 - Kraftmessdose.

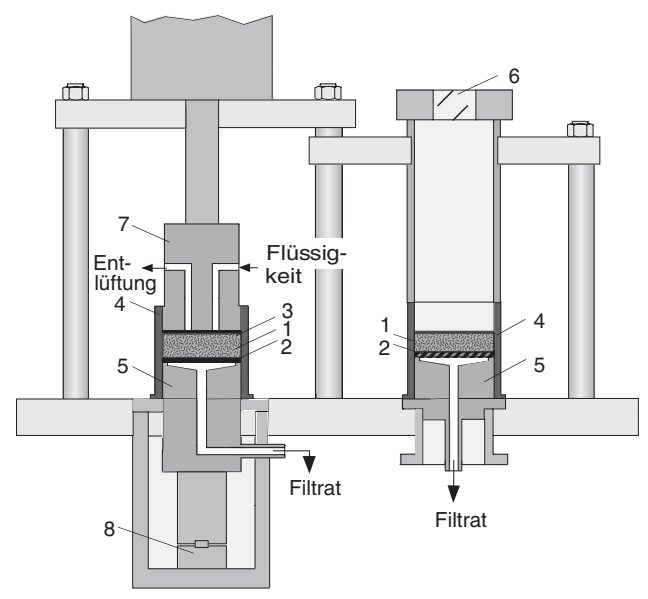

und über dem flächenbezogenen Filtratvolumen $v$ aufzutragen. Diese Auftragung erleichtert den Vergleich von Ergebnissen aus Versuchen mit unterschiedlichen Suspensionen, Filtrationsdrücken und Filtergeometrien.

Versuche mit ansteigenden Druckstufen bieten eine einfache Möglichkeit zu prüfen, ob tatsächlich ein ideal kompressibler Filterkuchen vorliegt. Die Annahme gilt dann als berechtigt, wenn der Stufenversuch die gleichen Ergebnisse liefert wie Referenzversuche bei entsprechenden konstanten Drücken (s. Abb. 4).

An der C-P-Zelle wurden Standardtests zur Erfassung der Porosität und des spezifischen Kuchenwiderstandes in Abhängigkeit vom Pressdruck durchgeführt. In Versuchsreihen mit ansteigendem Druck wurde die Probe bei jeder Druckstufe von einem Presskolben bis zum Konsolidierungsgleichgewicht gepresst und anschließend durchströmt. An die C-P-Ergebnisse wurden dann die Materialfunktionen $\varepsilon\left(p_{\mathrm{S}}\right)$ und $\alpha\left(p_{\mathrm{S}}\right)$ angepasst, die für die Simulation erforderlich sind.

Die Versuchsmaterialien wurden stellvertretend für Stoffklassen mit unterschiedlichen Kompressionsmechanismen ausgewählt: Kaolin als feindisperses, plättchenförmiges Tonmineral („HI spezial“, GEBRÜDER DoRfNER GMBH \& Co., Hirschau, Massendichte $\rho_{\mathrm{S}}=2600 \mathrm{~kg} / \mathrm{m}^{3}$, mittlere Partikelgröße $x_{50,3}=1,3 \mu \mathrm{m}$ ); Cellulose als biegsa-

Abbildung 4.

Filtration mit Ruß bei konstanten oder stufenweise gesteigerten Drücken, experimentelle Daten.

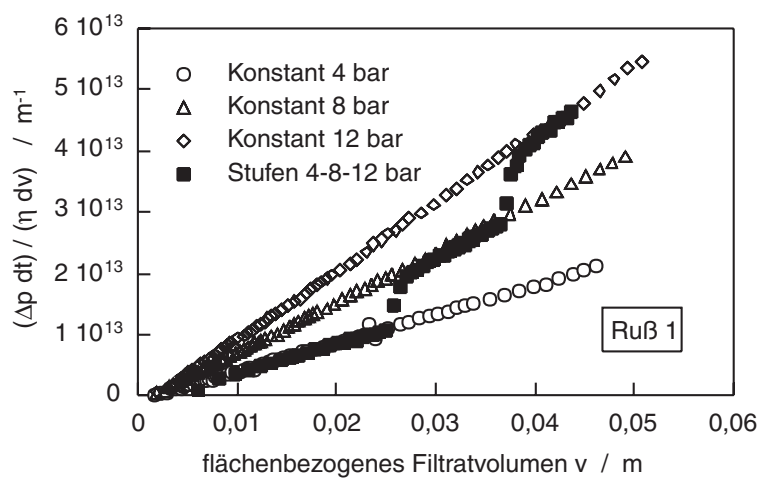


me Faser („Arbocel 600-30“, Schenk Filtertechnik GmbH, Waldstetten, $\left.\rho_{\mathrm{S}}=1820 \mathrm{~kg} / \mathrm{m}^{3}, \quad x_{50,3}=24,6 \mu \mathrm{m}\right) ; \quad$ elastisch deformierbare Partikel aus einem wasserunlöslichen Polymerisat („Divergan W“, BASF AG, Ludwigshafen, $\left.\rho_{\mathrm{S}}=1230 \mathrm{~kg} / \mathrm{m}^{3}, x_{50,3}=72 \mu \mathrm{m}\right), \mathrm{Ru}$ als nanoskaliges Pulver mit starker Agglomerationsneigung („FW 200“, DEGUSSA AG, Hanau, $\rho_{\mathrm{S}}=1870 \mathrm{~kg} / \mathrm{m}^{3}, x_{50,3}=13 \mathrm{~nm}$ ) und körniger Kalkstein als wenig kompressibles Referenzmaterial („MikroCalcilin“, Ulmer FÜLlstofF VertrieB, $\rho_{\mathrm{S}}=2700 \mathrm{~kg} / \mathrm{m}^{3}$, $\left.x_{50,3}=3,8 \mu \mathrm{m}\right)$. Rezepturen und Methoden zur Herstellung der wässrigen Suspensionen sind in [1] angegeben.

\section{Experimentelle Ergebnisse zum Materialverhalten}

Die C-P-Daten charakterisieren ein Stoffsystem wie ein Fingerabdruck. Die wichtigsten Kenngrößen sind Porosität und Widerstand im unbelasteten Zustand, $\varepsilon_{0}$ und $\alpha_{0}$, und die Exponenten der einfachen Potenzgesetze nach den Gln. (7) - (9), mit denen die Druckabhängigkeit von Porosität und Widerstand bereichsweise beschrieben werden kann. In Tab. 1 sind die Parameter der Materialgesetze für verschiedene Stoffsysteme zusammengefasst.

$$
\begin{array}{lll}
p_{\mathrm{S}} \leq p_{\mathrm{S}, \min }: & \alpha=\alpha_{0}, & \varepsilon=\varepsilon_{0} \\
p_{\mathrm{S}, \min }<p_{\mathrm{S}} \leq p_{\mathrm{S}, \mathrm{AB}}: & \alpha=\mathrm{a}_{\mathrm{A}} p_{\mathrm{S}}^{\mathrm{n}_{\mathrm{A}}^{*}}, & 1-\varepsilon=\mathrm{B}_{\mathrm{A}} p_{\mathrm{S}}^{\beta_{\mathrm{A}}^{*}} \\
p_{\mathrm{S}, \mathrm{AB}}<p_{\mathrm{S}}: & \alpha=\mathrm{a}_{\mathrm{B}} p_{\mathrm{S}}^{\mathrm{n}_{\mathrm{B}}^{*}}, & 1-\varepsilon=\mathrm{B}_{\mathrm{B}} p_{\mathrm{S}}^{\beta_{\mathrm{B}}^{*}}
\end{array}
$$

Die häufige Beobachtung, dass sich das Materialverhalten beim Überschreiten kritischer Drücke ändert, kann damit erklärt werden, dass in den einzelnen Druckbereichen verschiedene Kompressionsmechanismen dominieren. Alle untersuchten Materialien verhalten sich ideal kompressibel.

Filterkuchen aus dem elastisch deformierbaren Divergan sind bei Drücken unter 4 bar mäßig kompressibel, in diesem Bereich können sich die Partikel noch leicht umlagern. Bei höheren Drücken werden die Partikel mehr und

Tabelle 1.

\begin{tabular}{|c|c|c|c|c|}
\hline $\begin{array}{l}\text { Parameter / } \\
\text { Einheit }\end{array}$ & Kaolin & Divergan & Cellulose & Ruß 1 \\
\hline$p_{\mathrm{S}, \min } / \mathrm{Pa}$ & $3,52 \cdot 10^{3}$ & $1,56 \cdot 10^{4}$ & $2,11 \cdot 10^{4}$ & $1,77 \cdot 10^{4}$ \\
\hline$p_{\mathrm{S}, \mathrm{AB}} / \mathrm{Pa}$ & - & $4,26 \cdot 10^{5}$ & $5,43 \cdot 10^{5}$ & $9,84 \cdot 10^{4}$ \\
\hline$\alpha_{0} / \mathrm{m} \mathrm{kg}^{-1}$ & $3,70 \cdot 10^{11}$ & $3,68 \cdot 10^{10}$ & $5,83 \cdot 10^{10}$ & $5,25 \cdot 10^{11}$ \\
\hline$\varepsilon_{0} /-$ & 0,86 & 0,93 & 0,93 & 0,98 \\
\hline $\mathrm{n}_{\mathrm{A}}^{*} /-$ & 0,60 & 0,59 & 0,38 & 2,42 \\
\hline $\mathrm{n}_{\mathrm{B}}^{*} /-$ & - & 2,08 & 1,04 & 0,64 \\
\hline$\beta_{\mathrm{A}}^{*} /-$ & 0,16 & 0,33 & 0,43 & 0,95 \\
\hline$\beta_{\mathrm{B}}^{*} /-$ & - & 0,36 & 0,21 & 0,25 \\
\hline $\mathrm{a}_{\mathrm{A}} / \mathrm{m} \mathrm{kg}-1 \mathrm{~Pa}^{\mathrm{n}_{\mathrm{A}}^{*}}$ & $2,80 \cdot 10^{9}$ & $2,08 \cdot 10^{8}$ & $1,30 \cdot 10^{9}$ & 26,2 \\
\hline $\mathrm{a}_{\mathrm{B}} / \mathrm{m} \mathrm{kg}^{-} 1 \mathrm{~Pa}^{\mathrm{n}_{\mathrm{E}}^{*}}$ & - & 0,913 & $2,32 \cdot 10^{5}$ & $2,2 \cdot 10^{10}$ \\
\hline $\mathrm{B}_{\mathrm{A}} / \mathrm{Pa}^{\beta_{\mathrm{A}}^{*}}$ & $5,66 \cdot 10^{-2}$ & $4,22 \cdot 10^{-3}$ & $1,12 \cdot 10^{-3}$ & $3,16 \cdot 10^{-6}$ \\
\hline $\mathrm{B}_{\mathrm{B}} / \mathrm{Pa}^{\beta_{\mathrm{B}}^{*}}$ & - & $2,78 \cdot 10^{-3}$ & $1,85 \cdot 10^{-2}$ & $7,26 \cdot 10^{-3}$ \\
\hline
\end{tabular}

Parameter der Materialgesetze aus den Gln. (7) - (9) für verschiedene Stoffsysteme.
Abbildung 5.

Durchströmungswiderstand in Abhängigkeit des Feststoffgerüstdruckes für verschiedene Rußsuspensionen, experimentelle Daten.

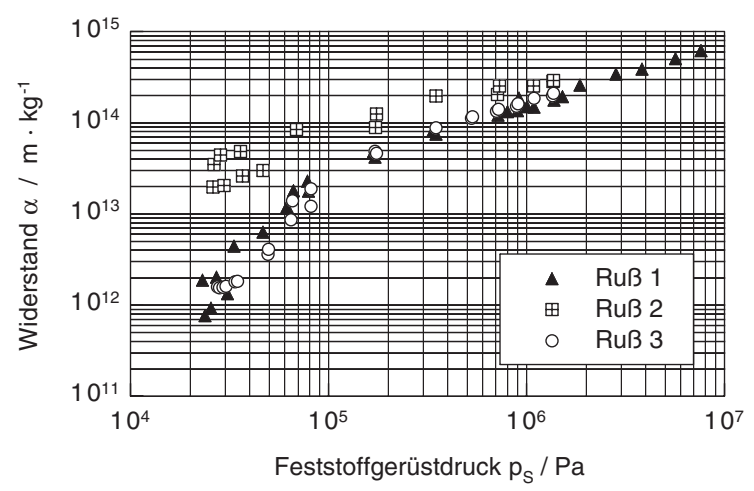

mehr zusammengequetscht, sie zwängen sich in immer engere Hohlräume. Der Widerstand nimmt rapide zu, die Filterkuchen sind super kompressibel. Ähnlich verhalten sich Filterkuchen aus biegsamen Cellulosefasern.

Aber auch starre Partikel können kompressible Haufwerke aufbauen. Plättchenförmige Kaolinpartikel lagern sich im unbelasteten Zustand in kartenhausähnlichen Strukturen an. Bei höheren Drücken klappen die Plättchen $\mathrm{zu}$ flachen Stapeln um und werden sukzessive weiter zusammengeschoben. Dies führt zu einer mäßigen Kompressibilität.

Die stärksten Veränderung des Widerstandes wurden an Filterkuchen aus agglomerierten nanoskaligen Rußpartikeln gemessen. An diesem Material lässt sich klar der Einfluss einer Suspensionsvorbehandlung demonstrieren. In Filterkuchen aus solchen feinen Partikeln liegen sehr enge Zwischenräume vor, so dass der Durchströmungswiderstand selbst bei hohen Porositäten groß ist. Wenn sich die Partikel in Agglomeraten (Flocken) zusammenlagern, entstehen gröbere Kanäle, durch die das Filtrat leichter abdrainieren kann. Dieser positive Effekt geht jedoch verloren, wenn die Agglomerate unter Druckbeanspruchung zusammengeschoben werden, der Widerstand steigt dann schnell auf den Wert des ungeflockten Systems (s. Abb. 5). Eine Suspensionsvorbehandlung führt also nur dann zum Erfolg, wenn der Filtrationsdruck auf die hohe Kompressibilität der Flocken abgestimmt wird.

\section{Prozessstrategien \\ 7.1 Wahl des Filtrationsdruckes bei der Kuchenbildung}

Für die Auslegung von Filtrationsprozessen ist der Filtrationsdruck der wichtigste Parameter, weil er das treibende Potential und damit die Geschwindigkeit der Kuchenbildung bestimmt. Hinzu kommt, dass die Auslegung und die Konstruktion eines Filterapparates auf den maximalen Filtrationsdruck abgestimmt werden müssen.

Die bei inkompressiblen Kuchen bewährte Maxime „viel hilft viel“ kann nicht ungeprüft auf Filtrationsprozesse mit kompressiblen Kuchen übertragen werden. Zwar wird auch hier das treibende Potential durch den Filtrati- 
onsdruck vorgegeben, zugleich bewirkt der Filtrationsdruck aber auch eine Kompaktierung des Kuchens und damit eine Erhöhung seines Durchströmungswiderstandes, was sich negativ auf die Geschwindigkeit der Kuchenbildung auswirkt.

In der Praxis ist bisher umstritten, welcher der beiden Effekte dominiert. Weit verbreitet ist die Auffassung, dass es einen optimalen Druck geben könnte, bei dem der Durchsatz ein Maximum erreicht [10]. Demnach würde bei niedrigen Drücken der beschleunigende Effekt der Potentialerhöhung überwiegen, wohingegen sich bei höheren Drücken der negative Kompaktierungseffekt durchsetzen würde. Dieser These wurde mit rigorosen Modellrechnungen und experimentellen Untersuchungen nachgegangen [1].

Die theoretischen Betrachtungen zeigen, dass der druckabhängige Filtratstrom $q(\Delta p)$ stark mit der Kompressibilität variiert. Nur bei inkompressiblen Kuchen $(\mathrm{n}=0)$ wächst der Filtratstrom direkt proportional mit dem Druck. Bei kompressiblen Kuchen ( $\mathrm{n}>0$ ) ist das Wachstum degressiv. Mit steigender lokaler Kompressibilität $\mathrm{n}$ verlaufen die Kurven immer flacher. Für alle Werte von $\mathrm{n}>1$ wächst der Filtratstrom einem Grenzwert für unendlich hohe Drücke entgegen. Bei endlichen Drücken wird dieser Wert nicht erreicht, dennoch ist er ein wertvoller Orientierungswert für die Auslegung in der Praxis. Je kompressibler der Filterkuchen ist, desto niedriger liegt der Grenzwert und um so schneller kommt der Filtratstrom in seine Nähe. Hoch kompressible Filterkuchen reagieren schon bei sehr niedrigen Drücken kaum noch auf eine Drucksteigerung.

Diese Aussagen gelten selbst für den extremen Fall, dass die Kuchenstruktur beim Übertreten eines kritischen Druckes schlagartig kollabiert und damit ihr Widerstand drastisch zunimmt. Der Anstieg des Filtratstromes $q(\Delta p)$ wird dann stark gebremst, ein Absinken des Filtratstroms tritt jedoch bei ideal kompressiblen Materialien in keinem Fall auf (s. Abb. 6). Demnach gibt es auch bei höchst kompressiblen Materialien keinen optimalen Druck.

Das degressive Ansteigen des Filtratstroms mit zunehmendem Druck wird ebenso wie die Stagnation des Filtratstroms bei hohen Drücken vielfach beobachtet [27, 28]. Die eigenen experimentellen Ergebnisse bestätigen diese Aussagen. Entsprechend dem Kompressibilitätsgrad der Materialien verhalten sich die Kurven für den Filtrat-

Abbildung 6.

Filtratstrom in Abhängigkeit vom Filtrationsdruck bei Variation des Exponenten n, normierte Darstellung mit Bezugspunkt $q_{0}\left(p_{\mathrm{s}, 0}\right)$, Grundlagen der Berechnung in [1].

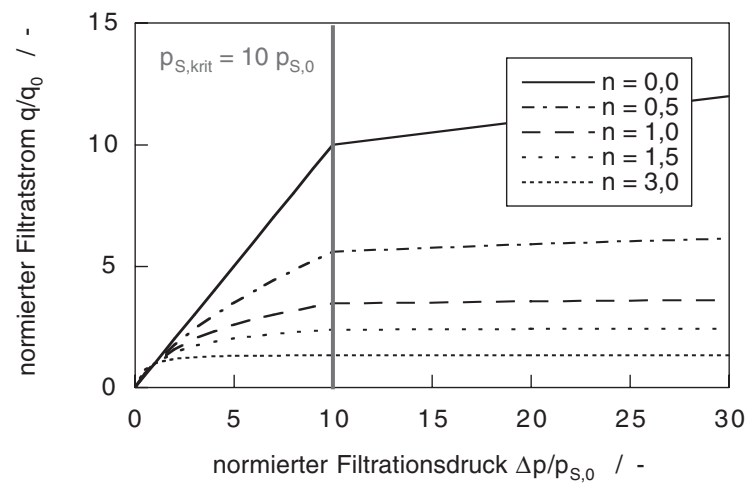

strom in Abhängigkeit des Filtrationsdrucks. Die Kurve von Micro-Calcilin $\left(\mathrm{N}_{\alpha}=0,18\right)$ kommt der Grenzkurve für den inkompressiblen Fall am nächsten. Bei Kaolin $\left(\mathrm{N}_{\alpha}=0,38\right)$ wächst der Filtratstrom deutlich degressiv mit dem Druck, bei Ruß $\left(\mathrm{N}_{\alpha}=0,78\right)$ stagniert er praktisch auf niedrigem Niveau. Ein optimaler Druck tritt bei keinem der Materialien auf [1].

Bei nicht ideal kompressiblen Materialverhalten, sei es bedingt durch Nachrücken von Feinkorn, Verkleben von Poren, Kristallisationsvorgänge etc., wird der Widerstand nicht allein durch die Kompressibilität vorgegeben. Vielmehr kommen weitere Effekte hinzu, die beispielsweise von der Zeit, vom durchgesetzten Filtratvolumen oder von der Durchströmungsgeschwindigkeit abhängen können. Wenn bei der Auswertung experimenteller Ergebnisse allein die Abhängigkeit zwischen Filtratfluss und Filtrationsdruckdifferenz betrachtet wird, gehen Informationen $\mathrm{zu}$ weiteren Einflussgrößen verloren. Stattdessen sollte stets kritisch überprüft werden, ob tatsächlich ideal kompressibles Materialverhalten vorliegt. Dazu sind beispielsweise stichprobenartige C-P-Versuche mit verschiedenen Ausgangskuchenhöhen und verschiedenen Durchströmungsgeschwindigkeiten geeignet.

Alle Aussagen zum optimalen Druck beziehen sich bisher auf die Filtration unter Differenzdruck ohne zusätzliche Feldkräfte. In diesem Fall ist die Kompaktierung ideal kompressibler Kuchen allein auf Strömungskräfte zurückzuführen. Bei der Filtration in Zentrifugen ergibt sich ein völlig anderes Bild. Durch die Massenkräfte wird der Filterkuchen am Außenradius, also am Filtermedium, weitaus stärker zusammengepresst, als es die Durchströmungskräfte allein bewirken könnten. Wird die Schleuderziffer über einen kritischen Wert erhöht, dann überwiegt der Effekt der Verdichtung durch die Massenkräfte, und der Filtratstrom nimmt ab [23]. So kann bei hoch kompressiblen Filterkuchen eine optimale Schleuderziffer mit maximalem Durchsatz auftreten, die auch als optimaler Druck aufgefasst werden kann.

Wenn es auch aus verfahrenstechnischer Sicht im Regelfall, d.h. bei der Differenzdruckfiltration mit ideal kompressiblen Materialien, keinen optimalen Druck gibt, so wird die Wahl des Filtrationsdruckes doch aus Gründen der Wirtschaftlichkeit zu einer Optimierungsaufgabe. Apparate, die für hohe Drücke ausgelegt sind, sind in der Anschaffung und im Betrieb spezifisch teurer als Apparate im Niedrigdruckbereich. Wenn dem erhöhten finanziellen Aufwand nur eine geringe Verbesserung des Verfahrensergebnisses gegenüber steht, ist es ökonomisch sinnvoller, einen niedrigen Filtrationsdruck zu wählen.

\subsection{Kombination von Kuchenbildung und Pressung}

Kompressible Filterkuchen können durch Nachpressen weitergehend entwässert werden. Unter mechanischer Last, die beispielsweise durch einen Presskolben, eine Presswalze oder ein Pressband aufgebracht werden kann, wird der Kuchen verdichtet, wobei die Flüssigkeit aus den Poren verdrängt wird. Dadurch wird die Restfeuchte des Kuchens 
gesenkt, ohne dass eine Untersättigung auftritt. Eine mechanische Flüssigkeitsabtrennung ist weitaus energieeffizienter als eine thermische. Daher ist es in der Regel empfehlenswert, kompressible Filterkuchen vor dem Trocknen auszupressen.

Das Nachpressen bewirkt eine Vergleichmäßigung der Kuchenstruktur [29]. Nach Ablauf der so genannten primären Konsolidierung liegt ein homogener Pressling vor. Die sekundäre Konsolidierung, die auf zeitabhängigen Kriechvorgängen beruht, macht sich erst bei langen Presszeiten, die in technischen Prozessen selten relevant sind, bemerkbar und soll deshalb hier vernachlässigt werden.

Zur Beurteilung der Nachverdichtung bietet es sich an, das Konsolidierungspotential $\Delta \varepsilon$ als Kenngröße einzuführen. Es resultiert aus der mittleren Porosität nach der Kuchenbildung, $\bar{\varepsilon}(\Delta p)$, abzüglich der Porosität nach der Pressung beim gleichen Druck, $\varepsilon_{\infty}$. Letztere lässt sich aus den C-P-Materialgesetzen ableiten, wenn der Feststoffgerüstdruck dem Konsolidierungsdruck gleich gesetzt wird.

$\Delta \varepsilon(\Delta p)=\bar{\varepsilon}(\Delta p)-\varepsilon_{\infty}(\Delta p)=\bar{\varepsilon}(\Delta p)-\varepsilon\left(p_{\mathrm{S}}=\Delta p\right)$

Strenggenommen ist das nach Gl. (10) definierte Konsolidierungspotential ein Grenzwert für unendlich lange Presszeiten. Dieses Potential lässt sich jedoch auch mit moderaten Presszeiten weitestgehend ausschöpfen [20].

Charakteristisch für hoch kompressible Haufwerke ist die starke Abnahme der Porosität mit steigendem Pressdruck. Zugleich bleiben die mittleren Porositäten nach der Kuchenbildung nahezu unabhängig vom Druck bei hohen Werten. Wie das Beispiel von Ruß (s. Abb. 7) zeigt, ergibt sich schon bei niedrigen Drücken ein beachtliches Konsolidierungspotential. Auch bei höheren Drücken ist durch Auspressen immer noch eine spürbare Entwässerung erreichbar. Je kompressibler ein Material, umso attraktiver wird das Nachpressen.

Während bei hoch kompressiblen Kuchen hohe Drücke zur Kuchenbildung weder im Hinblick auf die Restfeuchte noch auf die Filtrationsgeschwindigkeit nennenswerte Vorteile bringen, können hohe Pressdrücke für eine weitergehende Entfeuchtung durchaus lohnenswert sein. Deshalb ist in solchen Fällen eine zweistufige Prozessführung empfehlenswert: zunächst die Kuchenbildung bei niedrigem Druck, gefolgt von einer Pressung bei hohem Druck.

Abbildung 7.

Konsolidierungspotential von Ruß, experimentelle Daten.

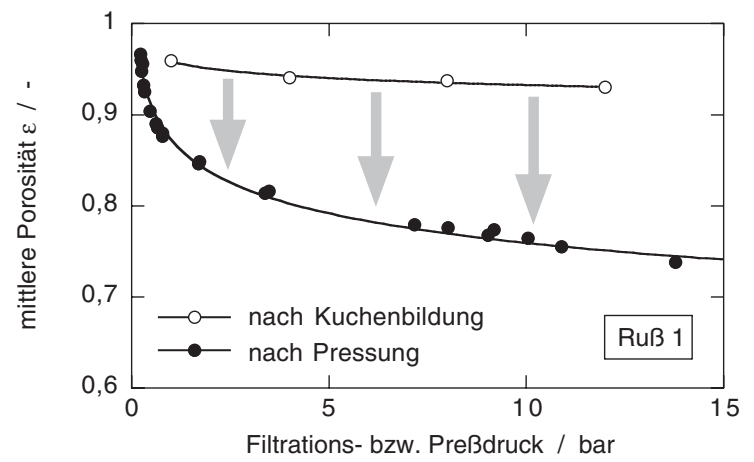

In vielen Prozessen mit kompressiblen Filterkuchen ist das Nachpressen notwendig, weil die inhomogene Struktur Probleme bei der Handhabung der feuchten $\mathrm{Ku}$ chen aufwirft, sei es beim Austrag aus dem Filter [2] oder bei der Weitergabe an einen Trockner [30].

Wenn der Filterkuchen gewaschen werden soll, ist es empfehlenswert, den Kuchen vor der Waschung zu pressen. Bei hoch kompressiblen Kuchen ist das Porenvolumen nach dem Pressen wesentlich geringer als unmittelbar nach der Kuchenbildung, dementsprechend wird weniger Flüssigkeit zum Auswaschen benötigt. Durch das Pressen wird die Porenradienverteilung im Filterkuchen eingeengt [31], was eine gleichmäßige Auswaschung begünstigt. Strukturänderungen, die während des Waschens aufgrund der Veränderung der physiko-chemischen Bedingungen im Filterkuchen auftreten können [32], machen sich an vorgepressten Kuchen weniger bemerkbar. Zwar müssen bei dieser Vorgehensweise längere Waschzeiten in Kauf genommen werden, weil der Pressling mit seiner kompakten Struktur einen höheren Durchströmungswiderstand als der ungepresste Filterkuchen hat, die Vorteile des Pressens überwiegen jedoch.

Für die Pressfiltration werden Apparate eingesetzt, in denen der mechanische Pressdruck über Kolben, Membranen oder Walzen aufgebracht wird. Leistungsfähige Pressfilter arbeiten mit vielen Entwässerungsflächen und geringen Kuchenhöhen. Auch in Filtern ohne spezielle Pressvorrichtung kann der Gasdifferenzdruck zum Nachpressen eingesetzt werden, wenn die Kuchenoberfläche durch eine feinporige Deckschicht oder eine gasundurchlässige Membran abgedeckt wird [2, 33, 34].

Pressfilter sind vergleichsweise aufwändige Apparate, die am effizientesten genutzt werden, wenn sie mit Schlämmen hohen Feststoffgehalts beschickt werden. Durch eine einfache Voreindickung im Erd- oder Zentrifugalfeld wird der Pressfilter spürbar entlastet. Hinzu kommt, dass die Presskinetik bei Schlämmen günstiger als bei Filterkuchen verläuft [29]. Beide Effekte tragen zur Einsparung an spezifisch teurer Pressfilterfläche bei.

\subsection{Wahl der Druckanstiegsfunktion}

In der Praxis ist es eine übliche Vorgehensweise, den Filtrationsdruck nicht schlagartig, sondern allmählich ansteigend aufzubringen. Dadurch soll eine frühzeitige Kompaktierung vermieden werden. Diese Methode wurde in Simulation und Experiment nachvollzogen [1].

Die Ergebnisse aus Experiment und Simulation zeigen übereinstimmend, dass die Kuchenbildung in allen Fällen bei einem konstanten hohen Druck früher zum Abschluss kommt als bei einer allmählichen Drucksteigerung bis zum gleichen Enddruck. Je später der Bereich niedriger Drücke verlassen wird, um so länger dauert die Kuchenbildung.

Diese Ergebnisse stehen im Einklang mit den Betrachtungen zur Wahl des Filtrationsdruckes. Für ideal kompressible Filterkuchen gibt es keinen optimalen Druck mit maximaler Kuchenbildungsgeschwindigkeit, sondern je 
nach Kompressibilität bewirkt eine Druckerhöhung eine mehr oder minder starke Beschleunigung der Filtration. Wenn der Filtrationsdruck erst allmählich gesteigert wird, verläuft die Kuchenbildung in der Anfangsphase langsamer als der Referenzvorgang, bei dem der Druck schon zu Beginn den Maximalwert annimmt. Dieser Nachteil kann nicht mehr wett gemacht werden. Der Zeitverlust ist bei inkompressiblen Kuchen, für die eine Drucksteigerung die stärkste Beschleunigung der Filtration bewirkt, am größten. Bei hoch kompressiblen Kuchen lässt sich die Filtrationsgeschwindigkeit durch eine Drucksteigerung nur geringfügig erhöhen. Ein mit der Zeit ansteigender Filtrationsdruck verursacht dann weniger schwerwiegende Einbußen bei der Filtrationsleistung.

Bei der Verwendung offenporiger Filtermedien kann es allerdings lohnenswert sein, den Druck allmählich aufzubauen, um die Bildung von Feststoffbrücken auf dem Filtermedium zu erleichtern. Bei zu hohen Anfangsdrücken besteht die Gefahr, dass die Brücken kollabieren und danach Partikel das Filtermedium verstopfen oder mit dem Filtrat ausgespült werden. Bei engporigen Filtermedien können von Anfang an hohe Filtrationsdrücke eingestellt werden, weil hier die Partikelabscheidung nicht auf Brückenbildung, sondern auf Sperreffekten beruht [1].

Weiterhin zeigt sich, dass die mittlere Porosität der Kuchen unabhängig von ihrer Vorgeschichte allein vom Enddruck abhängt. Dies legt den Schluss nahe, dass die Haufwerksstruktur allein von der momentanen Druckbelastung abhängt. Die Versuchsergebnisse in Abb. 6 belegen, dass sich bei einer stufenweisen Erhöhung des Filtrationsdrucks quasi spontan die gleichen Verhältnisse wie in den entsprechenden Referenzversuchen bei konstanten Drücken einstellen. Der Filterkuchen hat also kein „Gedächtnis“, d.h. er kann bei einer Drucksteigerung die durchlässigere Struktur aus zurückliegenden Phasen mit geringerer Druckbelastung nicht bewahren.

Ähnliches gilt für einen Druckanstieg bei der Pressfiltration: Die Konsolidierung kommt am schnellsten zum Abschluss, wenn der Pressdruck direkt nach der $\mathrm{Ku}$ chenbildung seinen Höchstwert erreicht [1, 29]. Das schlagartige Aufbringen des Pressdrucks kann allerdings dann Probleme aufwerfen, wenn die Abdichtung der Presskammer bei hohen Drücken den unverdichteten, fließfähigen Teil des Filterkuchens nicht zurückhalten kann. Derartige Schwierigkeiten können beispielsweise bei Siebbandpressen oder Plattenpressfiltern auftreten. In solchen Fällen empfiehlt es sich, den Pressdruck allmählich aufzubauen. Ein hinreichend vorverdichtetes Haufwerk lässt sich auch bei hohen Pressdrücken nicht mehr durch einen Dichtungsspalt quetschen.

\subsection{Intermittierende Kuchenfiltration}

Gerade bei kompressiblen Filterkuchen mit hohen Durchströmungswiderständen sind Prozessvarianten gefragt, die den Massendurchsatz wirksam erhöhen können. Eine einfache und zugleich sehr effiziente Methode dazu ist die intermittierende Kuchenfiltration (IKF) [1, 35]. Das Prinzip der IKF besteht darin, die Kuchenbildung bei kleinen Kuchen-

höhen zu unterbrechen, den Kuchen in der verbliebenen Suspension wieder anzumaischen und dann die Filtration fortzusetzen. Die Bildung hoher Filterkuchen mit entsprechend großen Widerständen wird also bis zum letzten Filtrationsschritt unterdrückt. Statt dessen wird die Anfangsphase der Kuchenbildung mit dem vergleichsweise hohen Filtratstrom mehrfach durchlaufen. Im Chargenbetrieb erhöht sich die Feststoffkonzentration in der Suspension bei jeder Resuspendierung, was wiederum zu einer Beschleunigung der Kuchenbildung führt.

Im Grenzfall ständigen Resuspendierens geht die IKF in die Querstromfiltration über, bei der die Kuchenbildung unterdrückt wird. Der Filterapparat wird dabei als Eindicker betrieben. Selbst wenn sich die Ablagerung von Feststoff am Filtermedium nicht vollständig vermeiden lässt, werden doch außerordentlich hohe Durchsätze erreicht.

Ergebnisse aus Simulation und Experiment demonstrieren die Leistungsfähigkeit der IKF [1]: Beim mäßig kompressiblen Kalkstein lässt sich die Filtrationszeit bei einmaligem Resuspendieren um über 20 \% verkürzen, beim ständigen Aufrühren beträgt die Zeitersparnis gegenüber der konventionellen Filtration ca. 60 \%. Beim hoch kompressiblen Ruß ist die Zeitersparnis noch beachtlicher: über $30 \%$ schon beim einmaligen Resuspendieren.

Somit stellt die IKF eine interessante Prozessvariante zur Leistungssteigerung dar, die sich mit geringem Aufwand in bestehende Prozesse integrieren lässt. Die IKF mit ständigem Resuspendieren wird als so genannte dynamische (Mikro-) Filtration in Scherspaltfiltern erfolgreich praktiziert. Konventionelle Filterapparate, wie z. B. Trommelfilter, Bandfilter, Kerzenfilter oder Rührwerksnutschen, können oft ohne große apparative Veränderungen zur IKF eingesetzt werden [1]. Damit ist insbesondere für hoch kompressible Filterkuchen eine einfache Möglichkeit gegeben, trotz hoher Filtrationswiderstände den Massendurchsatz wirksam zu erhöhen.

Besonderer Dank sei an dieser Stelle Herrn Prof. Dr.-Ing. WERNER STAHL ausgesprochen, von dessem unerschöpflichen Reichtum an wissenschaftlichen Ideen auch diese Arbeit wesentlich profitiert hat. Allen Studierenden und Mitarbeitern und Mitarbeiterinnen des Institutes für Mechanische Verfahrenstechnik und Mechanik an der Universität Karlsruhe (TH), die zum Gelingen dieser Forschungsarbeit beigetragen haben, gilt aufrichtiger Dank. Der Deutschen Forschungsgemeinschaft sei für finanzielle Unterstützung gedankt.

Eingegangen am 16. Juli 2003 [K 3268]

\section{Formelzeichen}

a

B

$h_{\mathrm{C}}$ $\mathrm{N}_{\alpha}$ $\mathrm{N}_{\varepsilon}$ $\mathrm{n}$

$\begin{array}{ll}{\left[\mathrm{m} \mathrm{kg}^{-1} \mathrm{~Pa}^{-\mathrm{n}^{*}}\right]} & \text { Koeffizient in Gln. (8), (9) } \\ {\left[\mathrm{Pa}^{-\beta^{*}}\right]} & \text { Koeffizient in Gln. (8), (9) } \\ {[\mathrm{m}]} & \text { Kuchenhöhe } \\ {[-]} & \text { Exponent in Gl. (4) } \\ {[-]} & \text { Exponent in Gl. (3) } \\ {[-]} & \text { Exponent in Gl. (6) }\end{array}$




\begin{tabular}{|c|c|c|}
\hline$n^{*}$ & {$[-]$} & Exponent in Gln. (8), (9) \\
\hline$P_{\mathrm{C}}$ & {$\left[\mathrm{m}^{-2}\right]$} & $\begin{array}{l}\text { längenspezifische Permeabilität des } \\
\text { Filterkuchens }\end{array}$ \\
\hline$p$ & {$[\mathrm{~Pa}]$} & Druck \\
\hline$p_{\mathrm{P}}$ & {$[\mathrm{Pa}]$} & Pressdruck \\
\hline$p_{\mathrm{S}}$ & {$[\mathrm{Pa}]$} & Feststoffgerüstdruck \\
\hline$P_{\mathrm{S}, 0}$ & {$[\mathrm{~Pa}]$} & Normierungsdruck \\
\hline$q$ & {$\left[\mathrm{~m} \mathrm{~s}^{-1}\right]$} & flächenspezifischer Volumenstrom \\
\hline$q_{0}$ & {$\left[\mathrm{~m} \mathrm{~s}^{-1}\right]$} & $\begin{array}{l}\text { flächenspezifischer Normierungs- } \\
\text { volumenstrom }\end{array}$ \\
\hline$R_{\mathrm{M}}$ & {$\left[\mathrm{m}^{-1}\right]$} & Durchströmungswiderstand des \\
\hline & & Filtermediums \\
\hline$r_{\mathrm{C}}$ & {$\left[\mathrm{m}^{-2}\right]$} & $\begin{array}{l}\text { längenspezifischer Durchströ- } \\
\text { mungswiderstand des Filterku- } \\
\text { chens }\end{array}$ \\
\hline$t$ & {$[\mathrm{~s}]$} & Filtrationszeit \\
\hline$v$ & {$\left[\mathrm{~m}^{-1}\right]$} & flächenspezifisches Volumen \\
\hline$w_{\mathrm{s}, \mathrm{c}}$ & {$\left[\mathrm{kg} / \mathrm{m}^{2}\right]$} & $\begin{array}{l}\text { flächenspezifische Kuchenfeststoff- } \\
\text { masse }\end{array}$ \\
\hline$x_{50,3}$ & {$[\mathrm{~m}]$} & $\begin{array}{l}\text { Medianwert der } Q_{3} \text {-Partikelgrößen- } \\
\text { verteilung }\end{array}$ \\
\hline$\alpha$ & {$\left[\mathrm{m} \mathrm{kg}^{-1}\right]$} & $\begin{array}{l}\text { massenspezifischer Durchströ- } \\
\text { mungswiderstand }\end{array}$ \\
\hline$\alpha_{0}$ & {$\left[\mathrm{~m} \mathrm{~kg}^{-1}\right]$} & $\begin{array}{l}\text { massenspez. Durchströmungswi- } \\
\text { derstand bei } p_{\mathrm{s}}=0\end{array}$ \\
\hline$\beta$ & {$[-]$} & Exponent in Gl. (5) \\
\hline$\beta^{*}$ & {$[-]$} & Exponent in Gln. (8), (9) \\
\hline$\Delta p$ & {$[\mathrm{~Pa}]$} & Druckdifferenz \\
\hline$\Delta p_{\mathrm{C}}$ & {$[\mathrm{Pa}]$} & Druckdifferenz am Filterkuchen \\
\hline$\Delta p_{0}$ & {$[\mathrm{~Pa}]$} & Normierungsdruckdifferenz \\
\hline$\varepsilon$ & {$[-]$} & Porosität \\
\hline$\varepsilon_{0}$ & {$[-]$} & Porosität bei $p_{\mathrm{s}}=0$ \\
\hline$\kappa$ & {$[-]$} & $\begin{array}{l}\text { Proportionalitätsfaktor, „Konzen- } \\
\text { trationsbeiwert“ }\end{array}$ \\
\hline$\eta$ & {$[\mathrm{Pa} \mathrm{s}]$} & $\begin{array}{l}\text { dynamische Viskosität der Suspen- } \\
\text { sionsflüssigkeit }\end{array}$ \\
\hline & {$\left[\mathrm{kg} \mathrm{m}^{-3}\right]$} & Massendichte des Feststoffes \\
\hline
\end{tabular}

\section{Literatur}

[1] C. M. Alles, Dissertation, Universität Karlsruhe (TH) 2000 (http://www.ubka.uni-karlsruhe.de/ cgi-bin/psview?document=2000/cheming/3).

[2] T. Wiedemann, Dissertation, Universität Karlsruhe (TH) 1996

[3] R. J. Wakeman, S. T. Thuraisingham, E. S. Tarleton, Filtr. Sep. 1989, (4), 277.

[4] W. Gösele, F \& S Filtr. Sep. 1995, (1), 14.

[5] J. Altmann, T. Weigert, S. Ripperger, F \& S Filtr. Sep. 1998, (5), 214.

[6] F. M. Tiller; C. S. Yeh, W. F. Leu, Sep. Sci. Technol. 1987, (2/3), 1037.
[7] H. Riemenschneider, Dissertation, Universität Stuttgart 1983.

[8] F. M. Tiller, AIChE J. 1973, (6), 1266.

[9] H. Anlauf, in Maschinen + Apparate zur Fest/Flüssig-Trennung, Grundlagen - Anwendung - Technik (Ed: W. Hess), Vulkan-Verlag, Essen 1991.

[10] B. Broens, H. Gasper, Handbuch der industriellen Fest/Flüssig-Filtration (Ed: H. Gasper), HüthigVerlag, Heidelberg 1990, 33.

[11] A. Rushton, A. S. Ward, R. S. Holdich, Solid-Liquid Filtration and Separation Technology, VCH-Verlag, Weinheim 1996.

[12] I. Nicolaou, Habilitation, Universität Karlsruhe (TH) 1996

[13] R. J. Wakeman, E. S. Tarleton, Filtration - Equipment Selection Modelling and Process Simulation, Elsevier Advanced Technology, Amsterdam 1999.

[14] H. Anlauf, F \& S Filtr. Sep. 1994, 2 (Teil 1), 63, 3 (Teil 2), 114.

[15] VDI-Richtlinie 2762, Verein Deutscher Ingenieure, Düsseldorf 1997.

[16] W. Bender, D. Redeker, Chem. Ing. Tech. 1981, 53 (4), 227-236

[17] F.M. Tiller, N. B. Hsyung, Water Sci. Technol. 1993, (1), 1.

[18] M. Shirato, M. Sambuichi, H. Kato, T. Aragaki, AIChE J. 1969, (3), 405.

[19] M. Zogg, Einführung in die Mechanische Verfahrenstechnik, B. G. Teubner, Stuttgart 1993.

[20] F. M. Tiller; C. S. Yeh, AIChE J. 1987, 3, 1241.

[21] D. X. He, R. B. H. Tan, C. Tien, Adv. Filtr. Sep. Technol. 1997, 11, 404.

[22] B. F. Ruth, Ind. Eng. Chem. 1946, (6), 564.

[23] T. Friedmann, Dissertation, ETH Zürich 1999

[24] F.M. Tiller, AIChE J. 1972, (1), 13.

[25] R.J. Wakeman, Trans. Inst. Chem. Eng. 1978, 56, 258.

[26] G. G. Chase, M. S. Willis, Chem. Eng. Sci. 1992, (6), 1373.

[27] F. M. Tiller; J. H. Kwon, AIChE J. 1998, (10), 1259.

[28] F. M. Tiller, W. P. Li, J. B. Lee, Water Sci. Technol. 2001, (10), 171.

[29] M. Shirato, T. Murase, M. Iwata, Progress in Filtration and Separation 4, (Ed: R. J. Wakeman), Elsevier, Amsterdam 1986, 181.

[30] M. Ruhland, Dissertation, Universität Karlsruhe (TH) 1999.

[31] P. Pfuff, Dissertation, Universität Karlsruhe (TH) 1992.

[32] J. Heuser, Dissertation, Universität Karlsruhe 2003.

[33] M. Shirato, T. Murase, E. Iritani, S. Nakatsuka, J. Chem. Eng. Japan 1985, (4), 372.

[34] W. Stahl, Verfahren zur Entfeuchtung eines Filterkuchens, Patentschrift DE 4123144 C1, Deutsches Patentamt, München 1992.

[35] F. M. Tiller, K.S. Cheng, Filtr. Sep. 1977, (1/2), 13. 